\title{
Extraordinary time-depended processes in the parametric interaction of counter-propagating waves
}

\author{
V.A.Tkachenko ${ }^{1}$, A.K. Popov $^{2}$, S.A. Myslivets ${ }^{3}$, V.V.Slabko ${ }^{1}$ \\ ${ }^{1}$ Institute of engineering physics and radio electronics, Siberian Federal University, Krasnoyarsk, Russia \\ ${ }^{2}$ Birck Nanotechnology Center, Perdue University, West Lafayette, USA \\ ${ }^{3}$ L.V. Kirensky Institute of Physics, Siberian Branch, Russian Academy of Sciences, RAS, Krasnoyarsk, Russia
}

\begin{abstract}
Three-wave mixing of ordinary and backward electromagnetic waves in the pulsed regime is investigated. It is shown that opposite direction of phase velocity and energy flux in the backward wave gives rise to extraordinary transient processes in the greatly enhanced optical parametric amplification and frequency-shifting nonlinear reflectivity. The discovered transients resemble time-delayed response of an oscillator on the pulsed excitation in the vicinity of its resonance.
\end{abstract}

Keywords - backward electromagnetic waves, transient parametric processes; counter-propagating waves; negative refractive index and negative dispersion.

Backward optical waves (BWs), the waves with contradirected wave and Poynting vectors, do not exist in natural material. However, they may appear in the metamaterials (MMs) with negative refraction index (NIMs). Coherent nonlinear optical propagation processes, such as harmonic generation and wave mixing, acquire extraordinary properties when one of the coupled waves is a BW. It is because energy flux of BW must be directed against others in order to achieve phase matching (Fig. 1a). Besides that, equal phase velocities of the coupled waves must be ensured, which presents a significant challenge. An approach alternative to NIMs was recently proposed $[1,2]$ that enables coexistence of ordinary and BWs which travel with equal phase velocities whereas their frequencies satisfy to the photon energy conservation (Fig. 1a). With such settings, optical parametric amplification (OPA) at $\omega_{2}$ dramatically changes from exponential $\exp (\boldsymbol{g} L)$ to $1 / \cos (\boldsymbol{g L})$, where $L$ is the metaslab thickness, and $g$ is proportional to strength of the pump field $\mathrm{S}_{3}$. and to the threewave mixing nonlinear susceptibility. At $g L \rightarrow j \pi / 2,(\mathrm{j}=1,3$, $5 \ldots)$, the output signal at $\omega_{2}$ and the idler at $\omega_{1}$ travelling in the reflection direction experience huge resonance enhancement (Fig. 1b).This gives rise to unparalleled transient processes.

For example, consider an ultimate case of semi-infinite pulse with sharp rectangular forefront and modules of group velocities for all wavesto be equal v. Two options are possible: continuous wave signal $\mathrm{S}_{2}$ and pulsed pump $\mathrm{S}_{3}$ and the opposite case of continuous wave $\mathrm{S}_{3}$ and pulsed $\mathrm{S}_{2}$. A set of coupled partial differential equations was solved numerically. We found that the shapes of forefronts of the output amplified and reflected pulses are almost identical in the indicated cases. However, a stark difference is found between the case of threewave mixing (TWM) of only ordinary $\left(\mathrm{S}_{1}\right.$ is co-directed with others) and the case of TWM of ordinary and backward waves $\left(S_{1}\right.$ is contra-directed to others as in Fig. $\left.1 b\right)$. In the first case, the shape of the output and input pulses remains identical:sharp rectangular. In the second case (Fig. 2), it is fundamentally different.Figure $2 \mathrm{a}$ shows that the raise time for the pulse forefront may significantly exceed the travel time through the metaslab $\Delta=\mathrm{L} / \mathrm{v}$. It increases as intensity of the pump field approaches the resonance value.

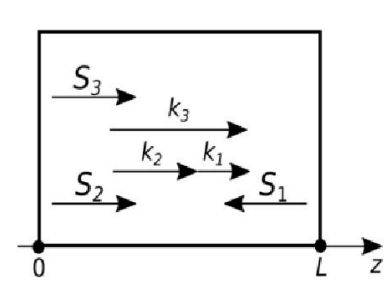

(a)

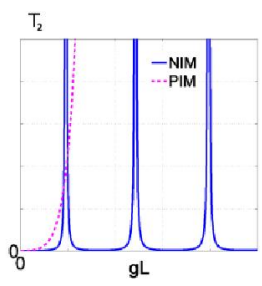

(b)
Fig. 1 (a) Three-wave mixing and phase matching of ordinary $\left(\mathrm{S}_{3}\right.$ and $\left.\mathrm{S}_{2}\right)$ and backward $\left(\mathrm{S}_{1}\right)$ waves. $\omega_{3}-\omega_{2}=\omega_{1} \cdot \mathrm{k}_{3}-\mathrm{k}_{2}=\mathrm{k}_{1}$. (b) "Geometrical" resonances in the dependence of the amplified transmitted signal $\mathrm{S}_{2}$ on intensity of the pump field $S_{3}$ in the continuous-wave regime. The dotted line corresponds to TWM of only ordinary waves.

A shape of the output pulses can be approximated as $T_{2}=A\left(1-\exp \left[\left(t-t_{0}\right) / \tau\right]\right)^{2}$. Here, $\mathrm{t}_{0}=\Delta \mathrm{t}$ and the transient period $\tau$ increases in the vicinity of the intensity resonance as shown in Fig. 2b. Additional information can be found in ref.[3].

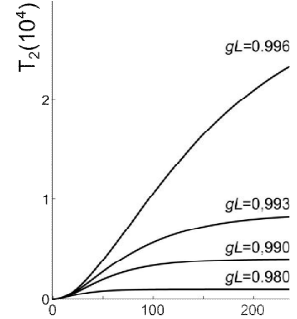

(a)

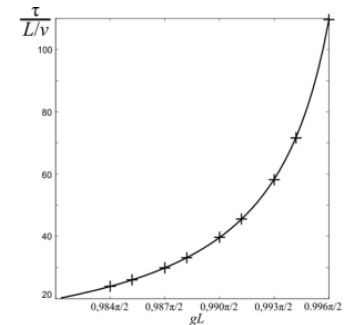

(b)
Fig. 2 (a) Time dependence of the output amplified signal. (b) Raise time $\tau$ vs. detuning from the resonance value of intensity of $S_{3}$.

Thus, herewith, we report extraordinary transient processes in optical parametric amplification and in the frequency-shifted nonlinear optical reflectivity, which originate from coherent nonlinear optical coupling of ordinary and contra-propagting backward travelling electromagnetic waves.

[1] A.K. Popov, M.I. Shalaev, S.A. Myslivets, V.V. Slabko, and I.S. Nefedov, "Enhancing coherent nonlinear-optical processes in nonmagnetic backward-wave materials," Appl. Phys. A vol. 109, pp. 835--840 (2012), doi:10.1007/s00339-012-7390-8.

[2] A.K. Popov, I.S. Nefedov, and S.A. Myslivets, "Phase matched backward-wave second harmonic generation in a hyperbolic carbon nanoforest," arXiv:1602.02497. 
[3] V.V. Slabko, A.K. Popov, V.A. Tkachenko, at al., "Transient processes in the parametric interaction of counter-propagating
waves"QuantumElectronics, vol.45(12), pp. 1151-1152, 2015. 